\title{
Nonequilibrium diffusion of reactive solid islands
}

\author{
F. Leroy, ${ }^{1, *}$ Y. Saito, ${ }^{2}$ F. Cheynis, ${ }^{1}$ E. Bussmann, ${ }^{3}$ O. Pierre-Louis, ${ }^{4}$ and P. Müller ${ }^{1}$ \\ ${ }^{1}$ Aix-Marseille Université, CINaM UMR 7325, Campus de Luminy, Case 913, F-13288 Marseille Cedex, France \\ ${ }^{2}$ Department of Physics, Keio University, 3-14-1 Hiyoshi, Kohoku-ku, Yokohama, Japan \\ ${ }^{3}$ Sandia National Laboratories, Albuquerque, New Mexico 87185, USA \\ ${ }^{4}$ Institut Lumière Matière, UMR 5306 Université Lyon 1-CNRS, Université de Lyon 69622 Villeurbanne, France
}

(Received 11 December 2013; revised manuscript received 20 May 2014; published 9 June 2014)

\begin{abstract}
We report on the observation of the random walk of solid-state silicon islands on $\mathrm{SiO}_{2}$ substrates during annealing at high temperatures. The mean square displacement (MSD) of the islands exhibits three regimes. At short times, the islands undergo equilibrium diffusion and begin to etch the surface thereby creating ringlike trenches. Then, an unusual size independent diffusionlike behavior is observed with a linear increase of the MSD. This behavior is attributed to a pinning instability of the triple line. Finally, as etching proceeds pits are formed in the substrate, and the MSD saturates as the islands are self-trapped in their own pits. Kinetic Monte Carlo simulations reproduce the main features of the three regimes, and provide a consistent picture of the microscopic reaction mechanisms at play in the experiments.
\end{abstract}

DOI: 10.1103/PhysRevB.89.235406

PACS number(s): 68.05.-n, 47.61.-k, 68.03.Cd, 83.50.Lh

Nanoparticles exhibit a large surface-to-volume ratio, leading to exacerbated interaction with their environment. For instance, the reactivity of nanoparticles is strongly enhanced, leading both to major environmental issues and to promising technological strategies. It is therefore important to understand the mechanisms involved in nanoparticle reactivity. Chemical reactions are actually known to drive nanoparticles towards a variety of nonequilibrium behaviors, such as self-propelled motion [1,2], or the formation of ring-shape trenches on solid substrates [3-6]. These behaviors are usually not specific to nanoscale systems, and are similar to those observed with macroscopic particles. For example, the reaction-mediated spontaneous drift of millimeter-scale solid-state camphor particles on the surface of water has been known for several centuries [7], and there is growing interest in artificial microswimmers in water, such as micrometer-size Pt Janus particles propelled by the dismutation of $\mathrm{H}_{2} \mathrm{O}_{2}$ [8]. Directed motion is also observed with liquid drops from micrometer to millimeter scales, e.g., with alcanes on glass [9], or $\mathrm{Ga} / \mathrm{GaAs}$ [10]. In addition, macroscopic reactive liquid droplets such as liquid $\mathrm{Si} / \mathrm{SiO}_{2}$ [4,11-13], or liquid metals on ceramic systems [14] exhibit complex pinning behavior at the liquid-solidvapor triple line. However, the physical processes at play in nanoparticle reactivity may differ from those of larger-scale objects. As an example, nanoparticles are known to exhibit larger thermal Brownian motion [15-20] and to suffer faster shape reorganization during annealing [21].

In this paper we report on the combined effects of reactivity, wetting, and shape changes on the random motion of crystalline $\mathrm{Si}$ islands on amorphous $\mathrm{SiO}_{2}$ substrates during annealing. For this purpose nanoparticle motion and size evolution are studied both experimentally (in situ and real time experiments) and theoretically [kinetic Monte Carlo simulations (KMC) including chemical reactivity). We show that the time dependence of the mean square displacement (MSD) of the islands successively exhibits three different

*leroy@cinam.univ-mrs.fr behaviors: (I) an equilibrium Brownian motion consistent with existing equilibrium theories [18,19], leading to strong size dependence of the diffusion constant, (II) an unexpected regime characterized by a linear time dependence of the MSD roughly independent on the nanoparticle size, attributed to a repeated pinning depinning of the triple line in ring-shape trenches formed by chemical reaction, and (III) a self-trapping regime where the reactive islands are trapped in the pit they drill in the substrate, leading to a saturation of the MSD. The experimental observations are supported by the KMC simulations, based on interface reaction of $\mathrm{Si}$ with $\mathrm{SiO}_{2}$, interface diffusion of oxygen, and evaporation of $\mathrm{SiO}$. They are in good agreement with the evolution of the interface morphology under the islands recently reported in [4].

The crystalline $\mathrm{Si}$ islands on amorphous $\mathrm{SiO}_{2}$ substrates are obtained by annealing at $1170 \mathrm{~K}$ a silicon-on-insulator (SOI) sample with a 22-nm-thick $\mathrm{Si}(001)$ film layer on top of an $\sim 140$-nm-thick silicon dioxide layer covering a $\mathrm{Si}(001)$ wafer, fabricated using the Smart-cut process at CEALETI (Grenoble France) and prepared in ultrahigh vacuum conditions as described in Ref. [22]. In this temperature range the spontaneous dewetting of the single crystalline Si thin film leads to the formation of self-organized 3D Si islands [23,24]. After $\mathrm{Si}$ islands formation at $\sim 1170 \mathrm{~K}$, the system is heated at higher temperature $\sim 1270 \mathrm{~K}$ to activate the Si reaction with the $\mathrm{SiO}_{2}$ layer. The islands motion is recorded in situ and in real time (rate: $1 \mathrm{~Hz}$ ) by low energy electron microscopy (LEEM). It is completed by ex situ analysis by atomic force microscopy (AFM).

Figure 1(a) shows a LEEM image of the SOI substrate after dewetting at $1170 \mathrm{~K}$. The $\mathrm{Si}$ islands (radius distribution $\sim 150 \pm 50 \mathrm{~nm}$ ) appear as black dots on the silicon dioxide and are aligned approximately in $\langle 100\rangle$ directions [24]. When the temperature increases in the range $1220-1320 \mathrm{~K}$, the islands move and shrink [Figs. 1(a)-1(c)]. The linear decrease of the projected area as a function of time [Fig. 1(c)] suggests a consumption rate of $\mathrm{Si}$ proportional to the linear dimension of the islands. It is therefore tempting to speculate that the reaction occurs at the triple line. Assuming a constant rate 
(a)

(b)
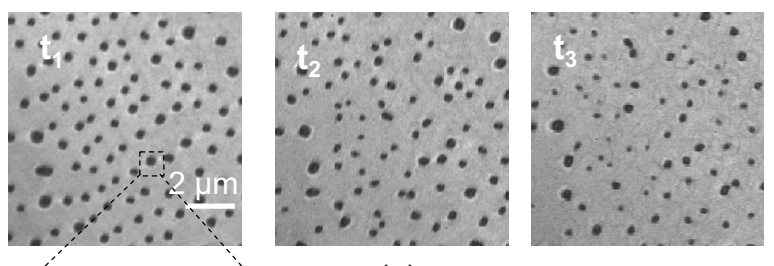

(c)
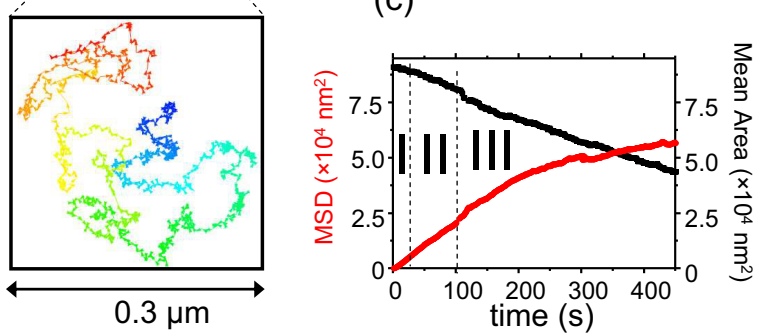

(d)

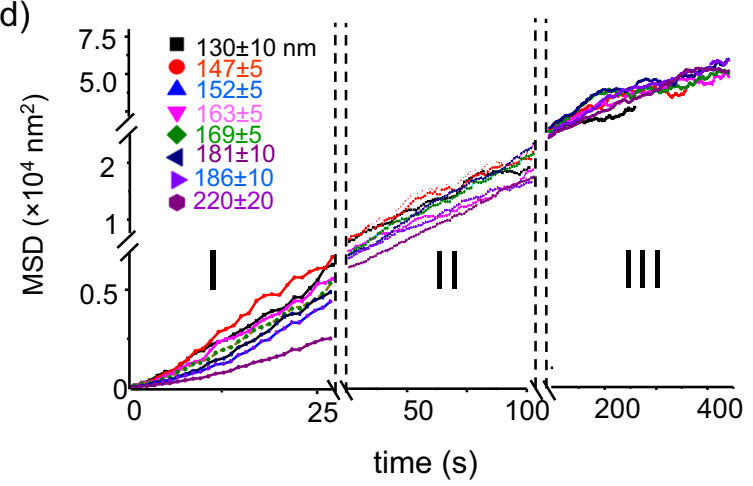

FIG. 1. (Color online) (a) LEEM images of $\mathrm{Si} / \mathrm{SiO}_{2}$ islands during annealing $(E=4.5 \mathrm{eV})$ at three different increasing times $\left(t_{1}=0\right.$ $\min , t_{2} \sim 15 \mathrm{~min}$, and $t_{2} \sim 30 \mathrm{~min}$ for $T \approx 1230 \mathrm{~K}$ ). The islands (diameter of $\sim 300 \mathrm{~nm}$ at $t_{1}$ ) move, shrink, and let fine visible tracks in the substrate $\left(t_{3}\right)$. (b) Example of trajectory of the center of mass of one Si nanoparticle. (c) Time evolutions of the mean square displacement (MSD) and mean projected area of $\sim 500 \mathrm{Si}$ islands under reaction ( $T \approx 1280 \mathrm{~K}$ ). (d) MSD for different radius of islands (average over 50 to $100 \mathrm{Si}$ islands) recorded in regimes I, II, and III.

$v_{\text {evap }}^{(3 \mathrm{D})}$ of reaction at the triple line and a truncated spherical shape of the islands, we obtain that the projected area $A$ obeys $\partial_{t} A=-4 a^{2} v_{\text {evap }}^{(3 \mathrm{D})} \sin ^{3} \theta /\left[(2+\cos \theta)(1-\cos \theta)^{2}\right]$, for a nanoparticle with contact angle $\theta$, and $a$ is an atomic scale. Using the experimental results $\partial_{t} A \approx-10^{2} \mathrm{~nm}^{2} \mathrm{~s}^{-1}$, with $\theta=$ $73^{\circ}[22,25]$, and $a \approx 0.3 \mathrm{~nm}$, we obtain $v_{\text {evap }}^{(3 \mathrm{D})} \approx 3 \times 10^{2} \mathrm{~s}^{-1}$ per atom along the triple line.

From the projected images of the $\mathrm{Si}$ islands we have also extracted the trajectories $\mathbf{r}(t)$ of the centers of mass of each nanoparticle over the entire process [26] [Fig. 1(b)]. We use the nanoparticle positions $\mathbf{r}(t)$ to calculate the MSD $\langle[\mathbf{r}(t)-$ $\left.\mathbf{r}(0)]^{2}\right\rangle$, where $t=0$ represents the beginning of annealing. The MSD exhibits three regimes as shown in Figs. 1(c) and 1(d).

A first linear regime, hereafter denoted as regime I, is found at short times. We extract the diffusion coefficient $D$ using the standard behavior of the MSD in the case of equilibrium Brownian motion $\left\langle[\mathbf{r}(t)-\mathbf{r}(0)]^{2}\right\rangle=4 D t$. We find that $D$ varies strongly with the nanoparticle size $D=D_{0} R^{-\alpha}$, where $R=(A / \pi)^{1 / 2}$ is the apparent radius and $\alpha=3.3 \pm 1$ [Fig. 2(a)]. The large experimental uncertainty on the $\alpha$ value originates from the short duration of the first regime that makes
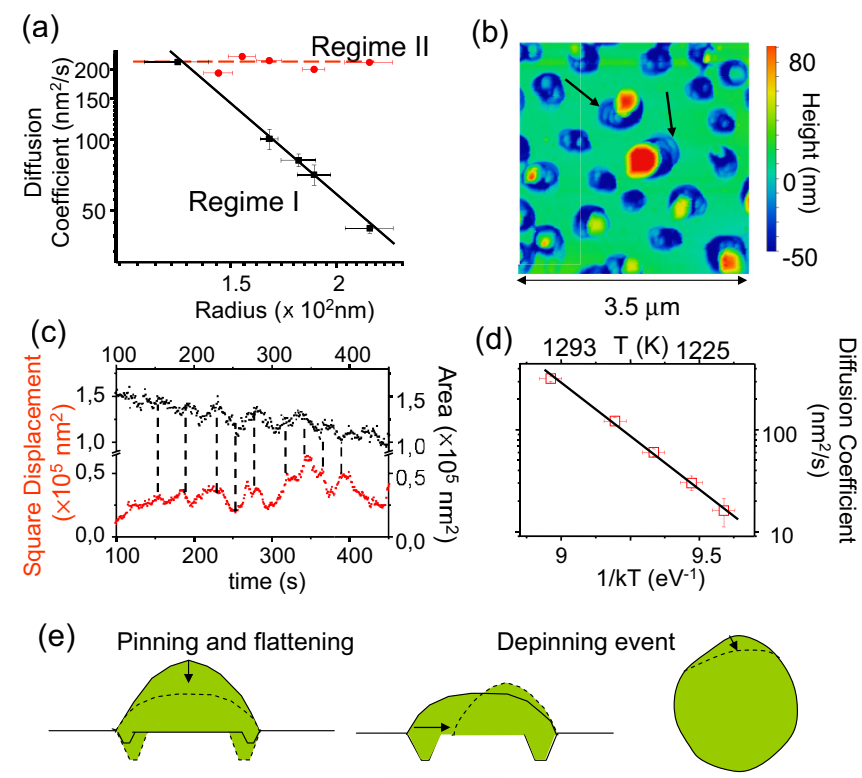

FIG. 2. (Color online) (a) Diffusion coefficient versus islands radius measured in the regimes I and II. (b) AFM image after partial reaction of the islands. Deep holes are observed corresponding to the complete consumption of the smallest islands. Reaction rings corresponding to pinning depinning mechanism are shown (arrows). (c) Projected nanoparticle area (black) and square displacement (red) variations in regime II. The in-phase correspondences are shown by vertical dash lines. (d) Diffusion coefficient versus temperature in regime II. (e) Sketch of the pinning-depinning cycles of the triple line associated to the reaction-induced morphology evolution.

a statistical analysis difficult. However, this result is consistent with the expected dependence of $D$ at equilibrium when the limiting mass transport mechanism is surface diffusion $\alpha=$ $d+1=4$, or surface attachment and detachment of atoms $\alpha=d=3$, where $d=3$ is the number of space dimensions [18]. However, because of the poor accuracy on the $\alpha$ value we cannot draw a conclusion on the main mechanism at work. Furthermore, we cannot exclude that both mechanisms occur simultaneously for reactive nanosized islands.

In the intermediate stages, denoted as regime II, the MSD exhibits a linear behavior [Fig. 1(c)]. However, we now find an effective diffusion coefficient $D_{\text {eff }}$ roughly independent on the nanoparticle size [Figs. 1(d) and 2(a)]. This size independence is inconsistent with all the equilibrium scenarios based on various microscopic mass transport mechanisms presented in the literature [18]. Previous works showed that interface alloying of $\mathrm{Sn}$ nanoparticles on $\mathrm{Cu}(111)$ substrates [1] produces a directed (ballistic) behavior at short times and a loss of velocity self-correlations at long times, also leading to increased diffusion. Here we claim that the physical origin of the phenomenon is completely different. We attribute the enhanced diffusion to a nonequilibrium effect, involving repeated pinning and depinning of the triple line. Indeed Fig. 2(c) shows for one nanoparticle the time evolution of its projected area and square displacement (SD). The nanoparticle area exhibits several oscillations that superimpose to the mean decay of the nanoparticle area due to its consumption. These area oscillations are well correlated to 
similar oscillations of the SD. In Fig. 2(c), a few in-phase oscillations are underlined. We interpret these correlations to pinning-depinning cycles of the triple line associated to the evolution of the interfacial morphology and shrinking of the nanoparticle [Fig. 2(e)]. This pinning-depinning process is also revealed by the presence of reaction rings in AFM images [Fig. 2(b); see also LEEM image on Fig. 1(a) at $t_{3}$ ]. The depinning leads to a sudden SD variation, whereas a new pinning event reduces temporarily its evolution. Studying various temperatures the effective diffusion coefficient of the islands can be described as a thermally activated process: $D_{\text {eff }}=\left(3 \pm 1 \times 10^{18} \mathrm{~nm}^{2} \mathrm{~s}^{-1}\right) e^{-\frac{(4.1 \pm 0.1 \mathrm{eV})}{k_{B} T}}$ [Fig. 2(d)]. It shows an activation energy of $E=4.1 \pm 0.1 \mathrm{eV}$ that largely exceeds the activation energy for $\mathrm{Si}$ adatom diffusion $E_{d} \sim 2.4 \mathrm{eV}$ [27]. Therefore, the pinning-depinning based diffusion process of the $\mathrm{Si}$ islands in regime II is kinetically controlled by the reaction involving $\mathrm{Si}$ and $\mathrm{SiO}_{2}$ substrate.

Finally, the MSD saturates until the islands shrink completely and disappear [Figs. 1(a)-1(c)]. We call this last stage regime III. This behavior is the opposite of that expected for nonreactive islands [28] as the diffusion coefficient should increase as the size of the islands decrease. As shown in Fig. 2(b), the reaction of the islands with the substrate leads to the formation of deep pits in the underlying substrate. It is therefore tempting to speculate that the saturation of the MSD is a consequence of the trapping of the islands in their own pits.

To clarify the microscopic origin of these regimes, we use a KMC model. We consider a two-dimensional heteroepitaxial solid-on-solid system on a square lattice. The lattice constant is set to unity. We use periodic boundary conditions along the $x$ direction, and the system is infinite in the $y$ direction. We consider a generic model with adsorbate atoms $\mathrm{A}$ and substrate molecules $\mathrm{S}$. On the column at $x=i$, substrate molecules $\mathrm{S}$ occupy sites from $y=-\infty$ to $y=h_{S}(i)$, and on top of them are adsorbate atoms A with a height $h_{A}(i)$. We assume first and second nearest-neighbor bonds between two A atoms, with energies $J_{A A 1}$ and $J_{A A 2}$, respectively. The ratio $\zeta=J_{A A 2} / J_{A A 1}$ controls the equilibrium shape of the nanoparticle $[29,30]$. A nanoparticle atom A also interacts with the first and the second nearest-neighboring substrate atoms $\mathrm{S}$ with interaction strengths, $J_{A S 1}$ and $J_{A S 2}$, respectively. We also choose $J_{A S 2} / J_{A S 1}=\zeta$. The ratio $\chi=J_{A S 1} / J_{A A 1}$ controls the wettability of the adsorbate to the substrate and $0<\chi<1$ favors the formation of 3D islands [30].

Since we expect no substrate surface diffusion of $\mathrm{SiO}_{2}$ at our annealing temperatures, the substrate molecules $\mathrm{S}$ are frozen. Surface diffusion of A atoms is implemented via the hops of the topmost $\mathrm{A}$ atom on one of neighboring columns. The types of bonds are indexed by $i=A A 1, A A 2, A S 1$, where $\mathrm{A}$ and $\mathrm{S}$ represent the particle and substrate atoms, respectively, and 1 or 2 represents nearest and next nearest neighbors, respectively. The hopping probability is $W_{A}=v_{A} \exp \left(-\sum_{i} n_{i} J_{i} / k_{B} T\right)$, where $v_{A}$ is a constant frequency, $k_{B}$ denotes the Boltzmann factor, $T$ is the temperature, and $J_{i}$ are the bonds to the atom before the hop. Chemical reactions are included by using four additional elementary processes. We first account for the $\mathrm{S} \leftrightarrow$ $\mathrm{A}+2 \mathrm{O}$ reaction: a $\mathrm{S}$ molecule $\left(=\mathrm{SiO}_{2}\right)$ at the interface may decompose to $\mathrm{A}(=\mathrm{Si})$ by emitting two $\mathrm{O}$ atoms. Denoting the associated energy change per bond as $\phi$, the rate of this process is $W_{D C}=v_{D C} e^{-m \phi / k_{B} T}$, where $v_{D C}$ is a constant frequency and $m$ is the number of lateral nearest-neighbor $\mathrm{S}-\mathrm{S}$ bonds before decomposition. The reverse oxidation rate is given as $W_{O X}=v_{D C}$. Then, following Refs. [4,31,32], we expect diffusion of $\mathrm{O}$ along the interface. The rates of oxygen diffusion along the $\mathrm{A} / \mathrm{S}$ interface and of evaporation of $\mathrm{A}$ and $\mathrm{O}$ atoms from a nanoparticle edge site are assumed independent of the local interface geometry for simplicity, and are set as $W_{O}=v_{O}$ and $W_{E}=v_{E}$.

Since the model has many parameters, we do not look for a systematic exploration of the parameter space, but we aim to show that the main features of the experiments can be
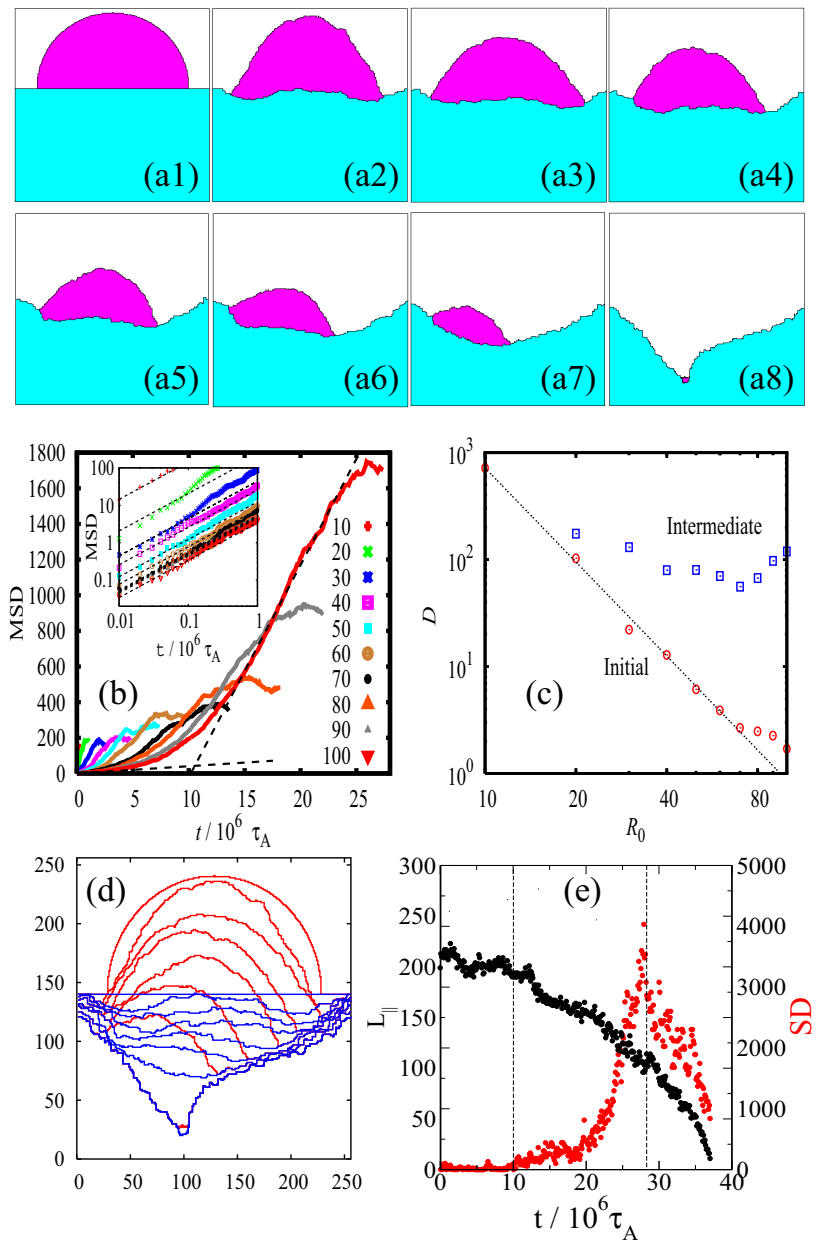

FIG. 3. (Color online) KMC simulations. (a) Snapshots for $t=$ $0,4.4 \times 10^{6}, 9 \times 10^{6}, 14 \times 10^{6}, 19 \times 10^{6}, 24 \times 10^{6}, 30 \times 10^{6}$, and $37 \times 10^{6}$ with initial radius $R_{0}=100$. In the initial stages, trenches appear near the triple line. In the final stages, a pit is formed. (b) MSD for different initial radii $R_{0}$, averaged over 100 simulations. Dashed lines are linear fits for short and intermediate times. Inset: linear behavior at short times. The dashed lines in the inset are linear fits. (c) Effective diffusion constant in the initial and intermediate regimes. The dashed line is a fit with $D \sim R_{0}^{-3}$. (d) Superimposed snapshots [(a1)-(a8)]. One may observe the initial depinning of the triple line on the right side, then the depinning of the triple line on the left side. (e) Distance $L_{\|}$between the triple lines and SD as a function of time. The times of the depinning events are indicated by dashed lines. 
recovered generically using our simplified picture. Hence we choose the parameters as follows: $k_{B} T=0.4 J_{A A 1}, \zeta=0.8$, and $\chi=0.5$, so that the equilibrium shape of a nanoparticle is almost isotropic and similar to a semicircle with a contact angle close to $90^{\circ}$, and $\phi=1.5 J_{A A 1}$ to keep the A-S interface smooth. We define the time $\tau_{A}=v_{A}^{-1} \exp \left[2(1+\zeta) J / k_{B} T\right]$ of detachment of an $\mathrm{A}$ atom from a kink on the $\mathrm{A}$ surface, and we choose $v_{O}=10 / \tau_{A}, v_{D C}=0.1 / \tau_{A}$, and $v_{E}=10^{-2} / \tau_{A}$, so that we have the time-scale hierarchy $v_{O} \gg 1 / \tau_{A} \gg v_{D C} \gg v_{E}$.

The results of the KMC model allow one to recover both the morphology and the random motion of islands observed in experiments. At all times, the evaporation rate of the nanoparticle is found approximately constant, with a weak decrease in the final stages. The number of atoms evaporated in the nanoparticle is therefore approximately $N(t)=N(0)-v_{\text {evap }}^{(2 \mathrm{D})} t$, with $v_{\text {evap }}^{(2 \mathrm{D})} \approx 5 \times 10^{-4} / \tau_{A}$. Assuming a roughly constant concentration $c_{O}$ of $\mathrm{O}$ atoms at the interface, we may assume that $v_{\text {evap }}^{(2 \mathrm{D})} \approx 2 v_{E} c_{O}$. The factor 2 comes from the fact that we have two triple points in 2D. Assuming local equilibrium, the concentration $c_{O}$ is determined from the balance of the reaction $\mathrm{S} \leftrightarrow \mathrm{A}+2 \mathrm{O}$, which reads $W_{O X} c_{O}^{2}=W_{D C}$, leading to $c_{O}=\exp \left[-(m / 2) \phi J / k_{B} T\right]$, where we set $m=2$ for an equilibration with the kinks. Using the values given above, we find $v_{\text {evap }}^{(2 D)}=4.6 \times 10^{-4} / \tau_{A}$, in good agreement with the simulation results.

As shown in Figs. 3(a2)-3(a4), and in agreement with experimental results shown in Fig. 2(b), trenches form in the vicinity of the triple line under the nanoparticle at short times in regime I. The inset in Fig. 3(b) indicates that the MSD behaves linearly at short times. From Fig. 3(c), one may extract a scaling behavior $D=D_{0} / R^{\alpha}$ with $\alpha \approx 2.92$ and $D_{0}=0.60 a^{2+\alpha} / \tau_{A}$ at short times. The exponent $\alpha \approx 3$ is in agreement with the value $\alpha=d+1$ expected for surface diffusion in thermodynamic equilibrium, where the space dimension is $d=2$. In addition, we expect $D_{0}=g(\theta) a^{5} / \tau_{A}$, where $g(\theta)=(\theta-$ $\sin \theta \cos \theta) /(\theta+\sin \theta \cos \theta)^{2}$, leading to $D_{0} \approx 0.64 a^{5} / \tau_{A}$ for $\theta=90^{\circ}$ in good agreement with the simulations.

In the intermediate stages, corresponding to regime II, we find a linear behavior of the mean-square displacement, as shown in Fig. 3(b). From this linear behavior, we extract an effective diffusion constant $D_{\text {eff }}$ using $\left\langle[\mathbf{r}(t)-\mathbf{r}(0)]^{2}\right\rangle=$ $A_{0}+4 D_{\text {eff }} t$ (the diffusion constant is difficult to measure for $R_{0}$ smaller than $\sim 20$ where regimes I and II are not well separated). Figure 3(c) shows that the effective diffusion constant exhibits a weaker dependence on the nanoparticle size than the early-time diffusion constant. This is again in agreement with experiments, as reported in Fig. 2(a).

Simulations on a single nanoparticle (without average) also reveal that one of the triple points is often pinned, while the other one recedes. As shown in Figs. 3(d) and 3(e), the depinning of the triple line leads to kinks in the time dependence of the SD of a nanoparticle, followed by a drift of the SD due to the shift of the nanoparticle center of mass. This observation is similar to the experimental results shown in Fig. 2(c). However, our simulations are not large enough to observe multiple pinning-depinning events. The pinning and depinning of the triple point also leads to an asymmetric position of the nanoparticle, which is again in agreement with the experiments [see Fig. 2(b)], where the islands are mostly found on the side of the holes (see also Ref. [4]).

In the final stages of the KMC simulations, we also observe a saturation of the MSD in Fig. 3(b), in agreement with experimental results shown in Fig. 1(c). In the very last stages, we clearly observed that the nanoparticle is trapped at the bottom of the pit that it has formed.

We have used a combination of real-time LEEM imaging and $\mathrm{KMC}$ simulations to show that reactive $\mathrm{Si}$ islands on $\mathrm{SiO}_{2}$ substrates exhibit a nontrivial diffusional behavior composed of three stages: (i) at short times, equilibrium diffusion; (ii) at intermediate times, nonequilibrium diffusion with an increased effective diffusion coefficient induced by the complex dynamics of the triple line; (iii) in the late stages, a mean-square displacement saturation due to trapping of the islands in the pits that they have formed. This last mechanism is the basis for technical applications such as the drilling of self-organized holes in $\mathrm{SiO}_{2}$ membranes [33,34].

We thank ANR 13 BS-000-402 grant LOTUS and JSPS KAKENHU 23540456.
[1] A. K. Schmid, N. C. Bartelt, and R. Q. Hwang, Science 290, 1561 (2000).

[2] M. L. Anderson, N. C. Bartelt, P. J. Feibelman, B. S. Swartzentruber, and G. L. Kellog, Phys. Rev. Lett. 98, 096106 (2007).

[3] U. Denker, O. G. Schmidt, N.-Y. Jin-Philipp, and K. Eberl, Appl. Phys. Lett. 78, 3723 (2001).

[4] K. Sudoh and M. Naito, J. Appl. Phys. 108, 083520 (2010).

[5] U. Denker, A. Rastelli, M. Stoffel, J. Tersoff, G. Katsaros, G. Constantini, K. Kern, N. Y. Jin-Phillip, D. E. Jesson, and O. G. Schmidt, Phys. Rev. Lett. 94, 216103 (2005).

[6] Y. Tu and J. Tersoff, Phys. Rev. Lett. 98, 096103 (2007).

[7] S. Nakata and Y. Hayashima, J. Chem. Soc., Faraday Trans. 94, 3655 (1998).

[8] J. Palacci, C. Cottin-Bizonne, C. Ybert, and L. Bocquet, Phys. Rev. Lett. 105, 088304 (2010).
[9] F. Domingues Dos Santos, and T. Ondarçuhu, Phys. Rev. Lett. 75, 2972 (1995).

[10] J. Tersoff, D. E. Jesson, and W. Tang, Science 324, 236 (2009).

[11] H. Kanai, S. Sugihara, H. Yamaguchi, T. Uchimaru, N. Obata, T. Kikuchi, F. Kimura, and M. Ichinokura, J. Mater. Sci. 42, 9529 (2007).

[12] D. Weiss, T. Gebensleben, L. Diestel, L. Alphei, V. Becker, and J. Becker, J. Mater. Sci. 46, 3436 (2011).

[13] D. Wachsmuth, T. Gebensleben, D. Weiss, V. Becker, L. Alphei, and J. Becker, J. Cryst. Growth 355, 122 (2012).

[14] O. Dezellus and N. Eustathopoulos, J. Mater. Sci. 45, 4256 (2010).

[15] H. Reiss, J. Appl. Phys. 39, 5045 (1968).

[16] A. Masson, J. J. Métois, and R. Kern, Surf. Sci. 27, 463 (1971).

[17] R. Kern, A. Masson, and J. J. Métois, Surf. Sci. 27, 483 (1971). 
[18] S. V. Khare and T. L. Einstein, Phys. Rev. B 54, 11752 (1996).

[19] J. M. Soler, Phys. Rev. B. 53, R10540 (1996).

[20] W. W. Pai, A. K. Swan, Z. Zhang, and J. F. Wendelken, Phys. Rev. Lett. 79, 3210 (1997).

[21] W. W. Mullins, J. Appl. Phys. 30, 77 (1959).

[22] E. Bussmann, F. Cheynis, F. Leroy, and P. Müller, IOP Conf. Ser. Mater. Sci. Eng. 12, 012016 (2010).

[23] E. Bussmann, F. Cheynis, F. Leroy, P. Müller, and O. PierreLouis, New J. Phys. 13, 043017 (2011).

[24] F. Cheynis, E. Bussmann, F. Leroy, T. Passanante, and P. Müller, Phys. Rev. B 84, 245439 (2011).

[25] D. T. Danielson, D. K. Sparacin, J. Michel, and L. C. Kimerling, J. Appl. Phys. 100, 083507 (2006).

[26] In order to avoid the consequences of a systematic drift, we use the average position of all the islands as a reference frame.
[27] M. E. Keeffe, C. Umbach, and J. Blakely, J. Phys. Chem. Solids 55, 965 (1994).

[28] Y. Saito, M. Dufay, and O. Pierre-Louis, Phys. Rev. Lett. 108, 245504 (2012).

[29] C. Rottman and M. Wortis, Phys. Rev. B 24, 6274 (1981).

[30] O. Pierre-Louis and Y. Saito, Eur. Phys. Lett. 86, 46004 (2009).

[31] R. A. B. Devine, D. Mathiot, W. L. Warren, D. M. Fleetwood, and B. Aspar, Appl. Phys. Lett. 63, 2926 (1993).

[32] R. A. B. Devine, D. Mathiot, W. L. Warren, and B. Aspar, J. Appl. Phys. 79, 2302 (1996).

[33] L. Borowik, J.-C. Barbé, E. Bussmann, F. Cheynis, F. Leroy, D. Mariolle, and P. Müller, US Patent 2012282758 (Nov. 8, 2012).

[34] L. Borowik, J.-C. Barbé, E. Bussmann, F. Cheynis, F. Leroy, D. Mariolle, and P. Müller, US Patent 2012282759 (Nov. 8, 2012). 